\title{
STUDIES IN METHIONINE AND SULFUR METABOLISM. I. THE FATE OF INTRAVENOUSLY ADMINISTERED METHIONINE, IN NORMAL INDIVIDUALS AND IN PATIENTS WITH LIVER DAMAGE ${ }^{1,2}$
}

\author{
By LAURANCE W. KINSELL, HAROLD A. HARPER, HARRY C. BARTON, \\ MAXINE E. HUTCHIN, AND JEAN R. HESS \\ (From the Division of Medicine, University of California Medical School, Department of \\ Biology, University of San Francisco, and Department of Medicine, U.S. \\ Naval Hospital; San Francisco and Oakland, California)
}

(Received for publication July 7, 1948)

The cardinal role of the liver in protein and amino acid metabolism has been well established. Consequently, studies of amino acid metabolism occupy a natural and fundamental place in an investigation of metabolic aberrations in hepatic disease. In 1913, Van Slyke and Meyer (1) demonstrated that certain amino acids when injected intravenously are rapidly removed from the circulation in the normal animal. They also demonstrated that a considerable capacity for temporary storage of amino acids existed in certain tissues, notably liver and muscle. Tolerance to various individual amino acids has been studied in connection with hepatic function. Thus Jastrowitz (2) in 1908 reported a greater urinary content of glycine in patients with liver damage than in normals following oral ingestion of glycine. A tolerance test to orally administered tyrosine was proposed by Bernhart and Schneider (3) as an aid to the diagnosis of hepatic disease. Similar procedures with single and multiple amino acid preparations have been studied by other investigators $(4,5)$. The recent development of specific and sensitive microbiologic procedures for the assay of amino acids (6) makes possible the further study of this problem.

By virtue of its prominent role in liver physiology, in the work here reported methionine was selected for a study of amino acid metabolism in normal and hepatopathic individuals. The presence of sulfur in the molecule confers an additional

\footnotetext{
1 This work has been performed under a contract between the Office of Naval Research and the University of California.

2 The biotin, pyridoxal, and pyridoxamine used in this study have been supplied through the courtesy of Merck \& Co., Inc.; the methionine, through the courtesy of Wyeth, Incorporated.
}

advantage since this serves as a "label" in the interpretation of the metabolic fate of the administered methionine.

In the present study a standart dose of $D L$ methionine was administered intravenously. $\mathrm{Ob}$ servations were then made on the rate of disappearance of the amino acid from the plasma together with a comparison of the excretion of free methionine and sulfate sulfur before and after the substance was given. Since the racemic $D L$ methionine was used, it was of interest to follow the metabolism of both isomers. This was made possible by the separate analysis of samples by two micro-organisms, one utilizing only the Lisomer, the other, both $D$ - and $L$-isomers.

\section{METHODS}

All patients (veteran and naval personnel) received a standard high protein, high carbohydrate intake over a two-day period preceding the test, during which time all medication was discontinued. They received no food from 8:00 p.m. the preceding night until after the 180minute blood and urine samples had been obtained. Methionine determinations were made on deproteinized filtrates of plasma obtained from heparinized blood After removal of a blood sample for determinations of the fasting methionine level, $50 \mathrm{cc}$. of a 3 per cent $D L$ methionine solution were injected over a five-minute period. Blood samples were then drawn at $15,30,60$, 120 , and 180 minutes after the injection. Urine samples corresponding to a two-hour period prior to the administration of the test dose of methionine as well as to the subsequent three-hour period were collected and assayed for methionine and, in some cases, sulfate sulfur (both free and total), and total amino nitrogen. This latter constituent will be discussed in a later communication. In several instances, methionine excretion during various periods up to 48 hours after the test dose had been given was measured.

Methionine was determined microbiologically using as test organisms Leuconostoc mesenteroides, P-60, for the assay of $L$-methionine and Lactobacillus fermenti, 36 , for 
assay of $D L$-methionine. Apparent $D$-methionine concentrations were obtained by difference. All other analytical details were as described in previous publications from this laboratory $(7,8)$. Standard chemical methods were used for the sulfur and amino acid determinations $(9,10)$.

\section{RESULTS}

The data here presented represent observations over a 15-month period on normal men and in men with acute, chronic, convalescent, and healed (inactive) liver disease.

\section{Fasting methionine values}

The plasma fasting $L$-methionine levels varied over a considerable range, from 0.22 to $1.48 \mathrm{mg}$. per cent in ngrmal controls with an average of $0.73 \pm 0.07^{3} \mathrm{mg} . / 100 \mathrm{cc}$. The average for pa-

$\therefore$ Standard error of the mean, $S_{x}=\sqrt{\frac{\Sigma d^{2}}{n(n-1)}}$. tients, if values at all stages of the disease are included, is higher than that of normals. Certain patients with very severe, active liver disease had extremely high fasting values, up to $4.00 \mathrm{mg}$. per cent, and an occasional patient who had received oral methionine in the recent past, had still higher plasma $L$-methionine levels; but over 90 per cent of the fasting values in patients fell in the control range. Except for those patients with very high values, no particular significance can be attached to the fasting levels, since a high degree of variability in fasting blood methionine is apparently the usual situation. Contrary to expectation, some individuals had measurable fasting levels of $D$ methionine.

\section{Observations on normal subjects}

Figure 1 illustrates the variations in plasma $L$ and $D$-methionine as observed at various intervals

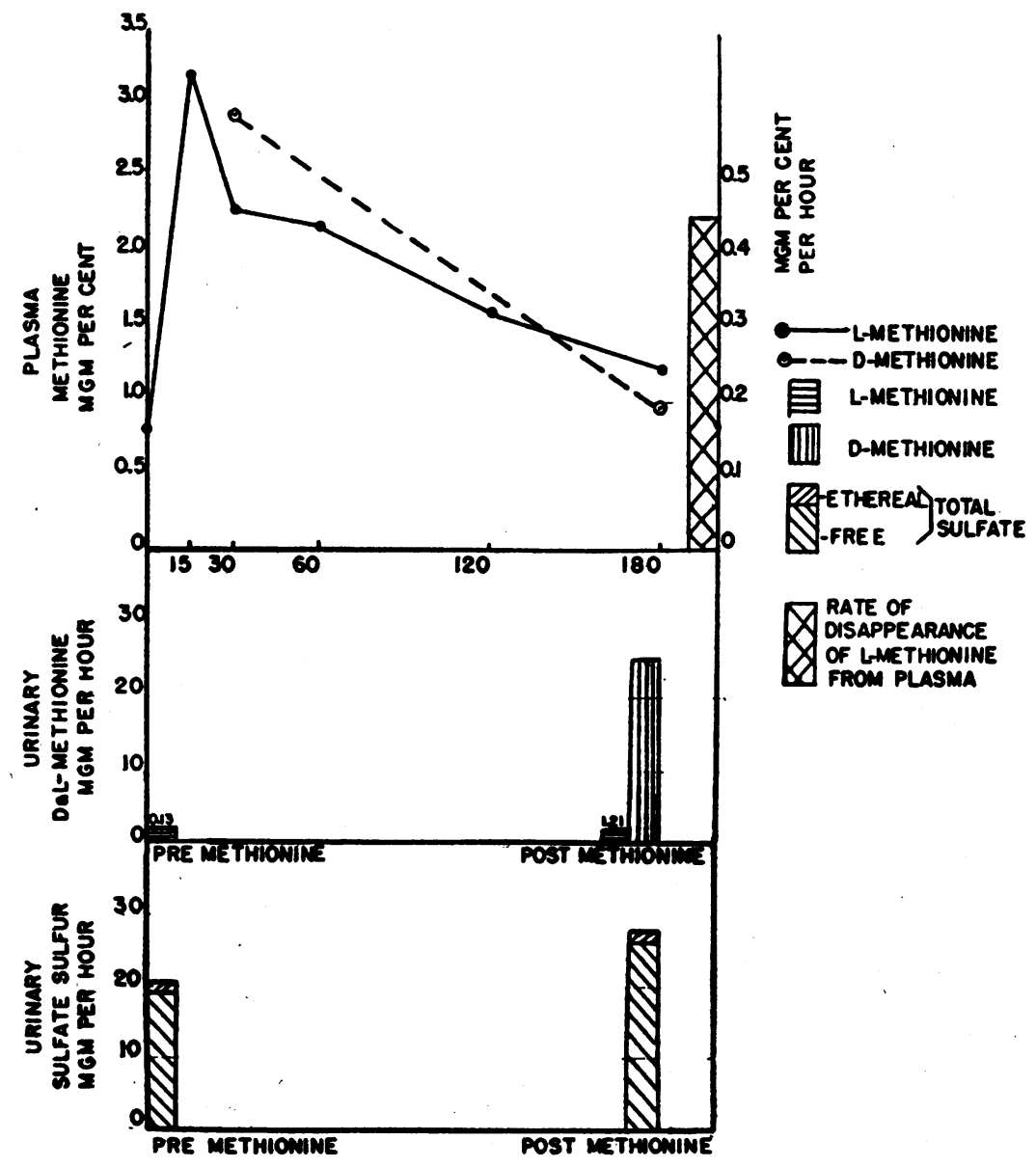

Fig. 1. Plasma and Urinary $D$ - and L-Methionine, and Urinary Sulfate, Prior and Subsequent to Methionine Infusion in Normal Individuals 

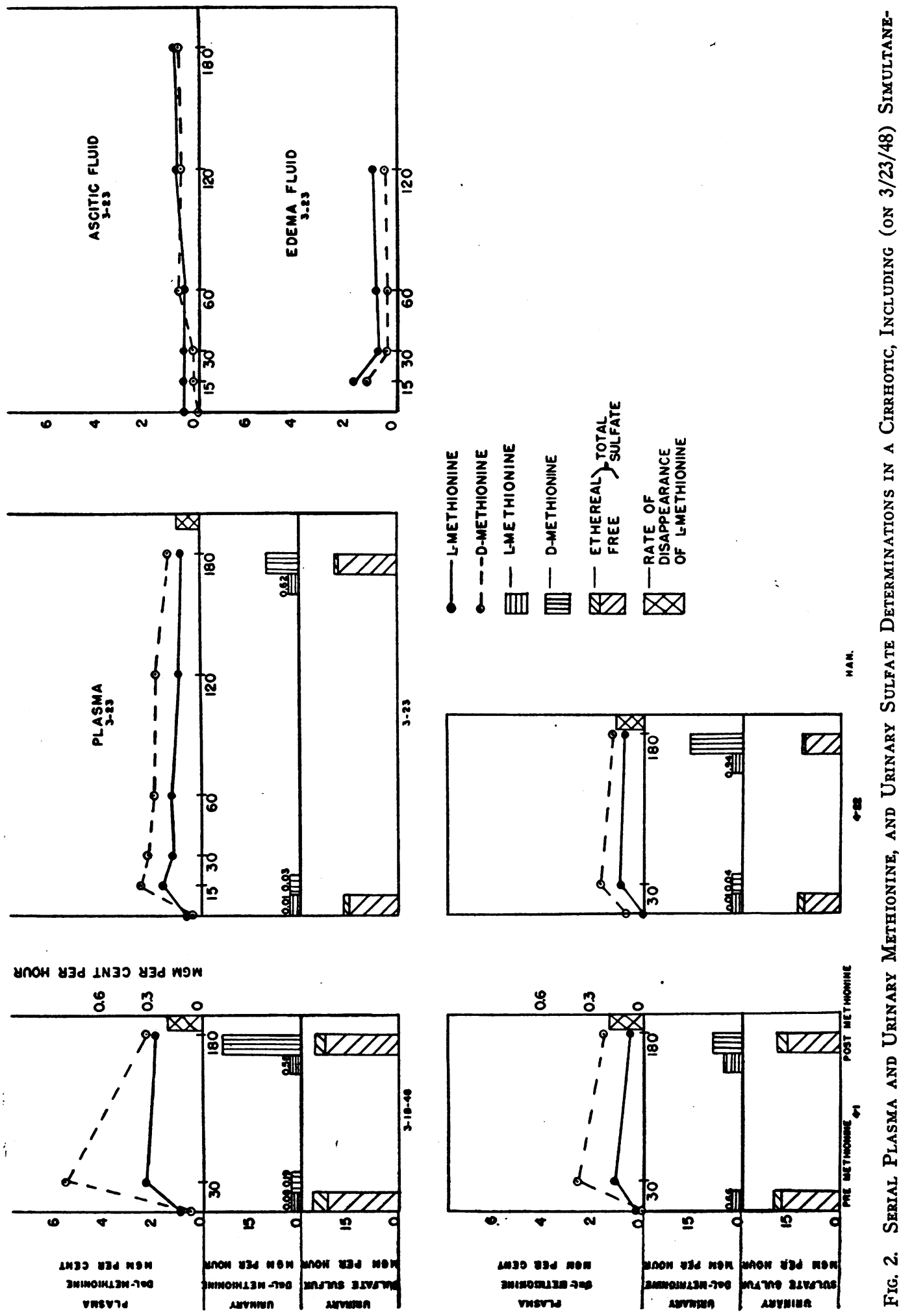
after the intravenous infusion of the methionine solution in normal subjects. It will be noted that the peak values obtained 15 minutes after the injection, decline rapidly up to the 30 -minute postinjection period, then more slowly and regularly over the succeeding 150 minutes. Apparently there is a very rapid initial diffusion of the amino acid into extravascular spaces, since even a very short time after the test dose was given, less than one-fourth of the injected material could be accounted for in the plasma. The rapid disappearance of $L$-methionine from the plasma during the 15 to 30 -minute period is presumably referable to diffusion plus utilization. The slow, steady

- drop in plasma $L$-methionine concentration over the 30 to 180 -minute period is attributed to utilization alone (anabolism plus catabolism). This interpretation is buttressed by observations on interstitial fluid methionine levels in a patient with cirrhosis (Figure 2). The actual figures obtained for the various points on the curve (in Figure 1) varied in different individuals since a fixed test dose was used regardless of variation in body size. However, by expressing the rate of disappearance as mg. of plasma methionine on a per hour basis, calculated for the $21 / 2$-hour period between the 30 - and 180-minute points, it was possible to reduce all studies to a comparable equivalent. In an original series of 11 normal individuals the average rate of disappearance was thus calculated to be $0.41 \pm 0.03 \mathrm{mg}$. per cent per hour for the $L$ isomer (7). The subsequent study of several other normals when added to this original series yielded a rate of $0.45 \pm 0.03 \mathrm{mg}$. per cent per hour. This latter figure has been used as an average normal standard in the comparative studies. of methionine metabolism in liver disease to be discussed in the present paper.

Figure 1 also details the average hourly excretion of $D L$ - and $L$-methionine as well as sulfate sulfur, total and ethereal, measured for the twohour period preceding the methionine injection and during the subsequent three hours. Negligible quantities of $L$-methionine were excreted at any time. After injection, a maximum of only 3.36 mg. per hour, with an average of $1.12 \pm .22 \mathrm{mg}$. per hour, was found. This indicates that even though the plasma $L$-methionine level was raised as much as five times the normal fasting level, the maximal rate of renal tubular reabsorption was not reached.

The curve illustrating the average $D$-methionine levels at intervals after injection of the test amino acid indicates that although the peak values are higher, those obtained at 180 minutes tend to be lower than the comparable $L$ values. Thus a higher rate of disappearance for the $D$-isomer was usually observed. Inspection of the data on rates and extent of excretion of the $D$-isomer shows that a very considerable quantity is excreted during the initial three hours and this fact probably accounts in large part for the more rapid removal of this isomer from the plasma. However, so far no consistent pattern for the $D$-isomer either in rates of disappearance from the plasma or excretion could be detected. Hence, only the $L$ form has been considered in evaluation of metabolic abnormalities in hepatic disease.

Urinary sulfate sulfur excretion during the two hours preceding and the three hours following methionine administration has been utilized as an index of the catabolism of endogenous, and exogenous-plus-endogenous sulfur-containing amino acids, respectively. In an initial group of six normal individuals an average pre-methionine rate of $20.3 \mathrm{mg}$. per hour was obtained, rising to a rate of $28.0 \mathrm{mg}$. per hour during the three-hour postmethionine period. Since the sulfur content of the $1500 \mathrm{mg}$. of methionine administered is 322 mg., it would seem that little of the methionine is catabolized during the initial three-hour period.

\section{Observations in liver disease}

It was anticipated that an acutely damaged liver could not as readily utilize administered methionine as the normal organ. The observations to follow support this hypothesis.

(a) Acute Hepatitis. Initial values from $14 \mathrm{pa}$ tients in the pre-convalescent phase of the disease are included in Figure 3. It will be noted that the average rate of disappearance of methionine from the plasma in these patients is slower than that for the normals. Ten of these patients had rates ranging from 0.13 to 0.26 with an average for all 14 patients of $0.26 \pm 0.02 \mathrm{mg}$. per cent. When compared to the average normal figure of $0.45 \pm 0.03$ 


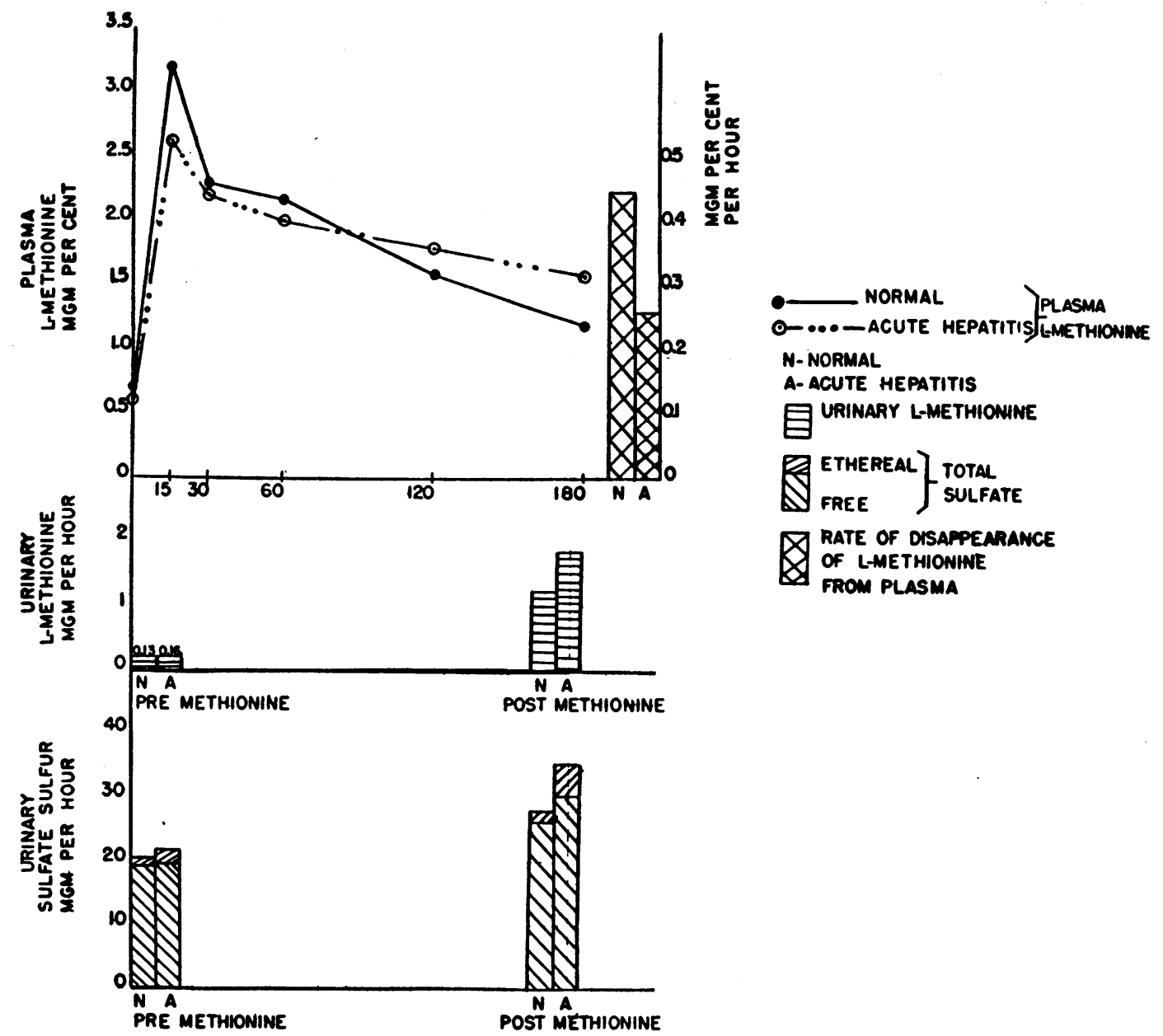

Fig. 3. Comparison of L-Methionine and Sulfate Values in Normal Individuals, and in Patients with Acute Hepatitis

mg. per cent, this difference was shown to be statistically significant $\left(D_{M}: S_{D_{M}}=4.7\right) .^{4}$

The urinary excretion of $L$-methionine after the test dose averages $1.83 \pm .52 \mathrm{mg}$. per hour as compared to $1.12 \pm .22$ in normals. For the twohour fasting period prior to the test dose, the hepatitis patients had an average excretion of $0.16 \mathrm{mg}$. per hour as compared to 0.12 in normals. Neither of these differences has statistical significance.

4This refers to the ratio of the difference of the means $\left(D_{M}\right)$ to the standard error of this difference $\left(S_{D_{M}}\right)$, calculated as follows: $S_{D_{M}} \sqrt{\frac{\Sigma d_{1}^{2}+\Sigma d_{2}^{2} / n_{1} n_{2}}{\left(n_{1}-1\right)\left(n_{2}-1\right) / n_{1}+n_{2}}}$, where $\Sigma d^{2}$ represents the sum of the squares of the differences from the mean and " $n$ " equals the number of cases. When this ratio exceeds 3 , results are considered significant.
Inorganic sulfate sulfur excretion in four of the acute hepatitis patients averaged $22.0 \mathrm{mg}$. per hour pre-methionine and $35.4 \mathrm{mg}$. per hour postmethionine. Insufficient data are at hand to permit of valid statistical evaluation at this time.

(b) Convalescent Hepatitis. As convalescence progresses in the patient with acute hepatitis, the rate of disappearance of administered $L$-methionine rises to normal or super-normal levels and the urinary sulfate excretion approaches the normal pattern. These findings are included in serial observations in a representative patient during the acute and convalescent phases of a moderately severe hepatitis (Figure 4). The clinical course of the disease is indicated by the total serum bilirubin levels. During the early acute phase of the disease, it will be noted that in addition to a minimal rate 
of disappearance of plasma $L$-methionine, the fasting $L$-methionine levels were markedly elevatedto more than four times the average fasting level. Return to a normal fasting level preceded the restoration of a normal rate of disappearance.

(c) Chronic Hepatitis. The diagnosis of chronic viral hepatitis is arbitrarily assigned to those patients who, over a period of six months or longer have failed to recover from an attack of established or presumptive hepatitis. In view of the obscure etiology of the syndrome and the likelihood of progressive hepatocellular damage and replacement fibrosis (cirrhosis) in such individuals, these patients represent a small but important segment of the entire group. In Figure 5, data on three such individuals are presented. The rate of disappearance of plasma methionine is normal in all but one instance. A high fasting methionine is noted on one occasion in the man with the most severe hepatitis together with a very high level of urinary sulfate, then and subsequently. The data in these patients is still inadequate for any but inferential purposes. A high rate of catabolism may explain the "normal" rates (vide infra).

(d) Chronic Liver Damage (Cirrhosis). The term "cirrhosis" is used in referring to patients under study in this group. All patients so designated have extensive hepatic fibrosis with or without fatty infiltration, with varying degrees of active hepatocellular damage. The exciting etiology in the majority of instances is chronic alcoholism with resultant and/or concomitant dietary insufficiency, continuing or recurring over a period of many years. Some of the patients have had



Fig. 4. Serial Evaluation of Methionine-Sulfur Metabolism during the Course of an Attack of Acute Hepatitis in Patient SMI

The clinical status of the disease is indicated by the serum bilirubin levels. 


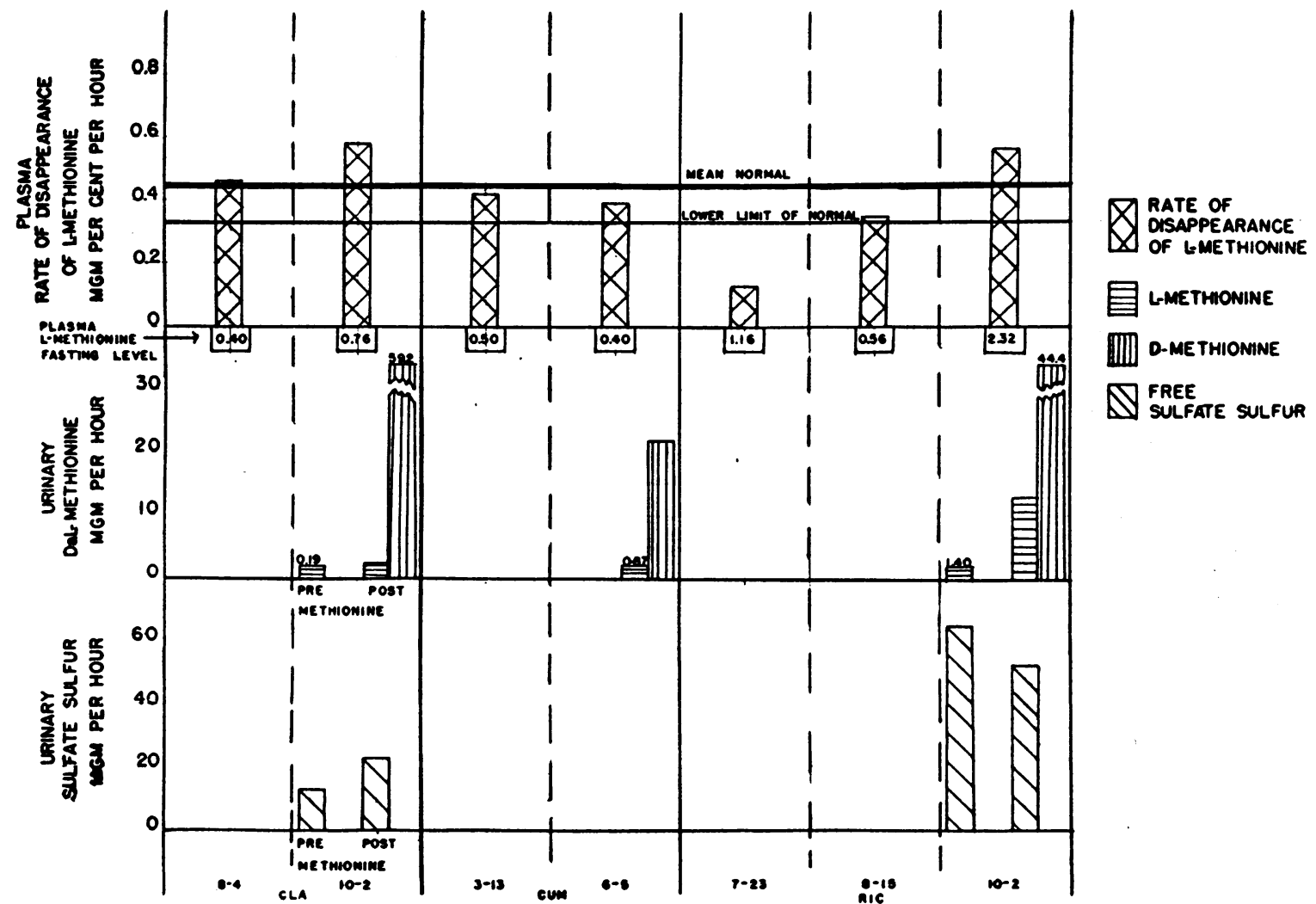

Fig. 5. Chronic Viral Hepatitis; Methionine and Sulfate Studies in Three Patients

marked degrees of ascites, with or without other manifestations of portal hypertension. The diagnosis has been proven in all instances by one or more biopsies. We regard these patients as being most satisfactory for controlled metabolic studies because of "known" etiology, absence of unpredictable fluctuation in clinical and biochemical status, and because of the existence of a "panel" of tests which roughly but adequately reflect the histological and physiological status of the liver.

The same procedures and technics were used as in the preceding groups of patients. Figure 6 shows the average values initially obtained in a group of ten such patients, in three of whom only fasting levels were obtained.

Considering only these determinations, an average rate of disappearance of $0.26 \pm 0.04$ was obtained. The difference between this initial rate and that already noted in normals is statistically significant $\left(D_{M}: S_{D_{M}}=3.2\right)$. When 26 values obtained in 12 patients at all stages of the disease were averaged, a mean rate of $0.22 \pm 0.05 \mathrm{mg}$. per
100 cc. per hour resulted. Statistical comparison of this figure with that obtained in normals reveals a ratio of $D_{M}$ to $S_{D_{M}}$ of 10 - a highly significant difference. The inclusion of only the initial values in construction of Figure 6 is done for the sake of uniformity; i.e., to permit of proper comparison between these data and those obtained in hepatitis patients.

$L$-methionine excretion averaged $0.163 \mathrm{mg}$. per hour before injection, and $0.770 \mathrm{mg}$. per hour post-injection, figures not significantly different from the control values. This statement also applies to values obtained in all stages of the disease.

The urinary sulfate values included in Figure 6 are too few for significance. In Figure 7 are shown a series of pre- and post-methionine urinary sulfate values obtained in a group of patients with chronic liver damage. It will be noted that the majority of these values fall within the normal range. Two highly abnormal values are included at this time only for purposes of brief comment (RIC and DRE). While both had high 


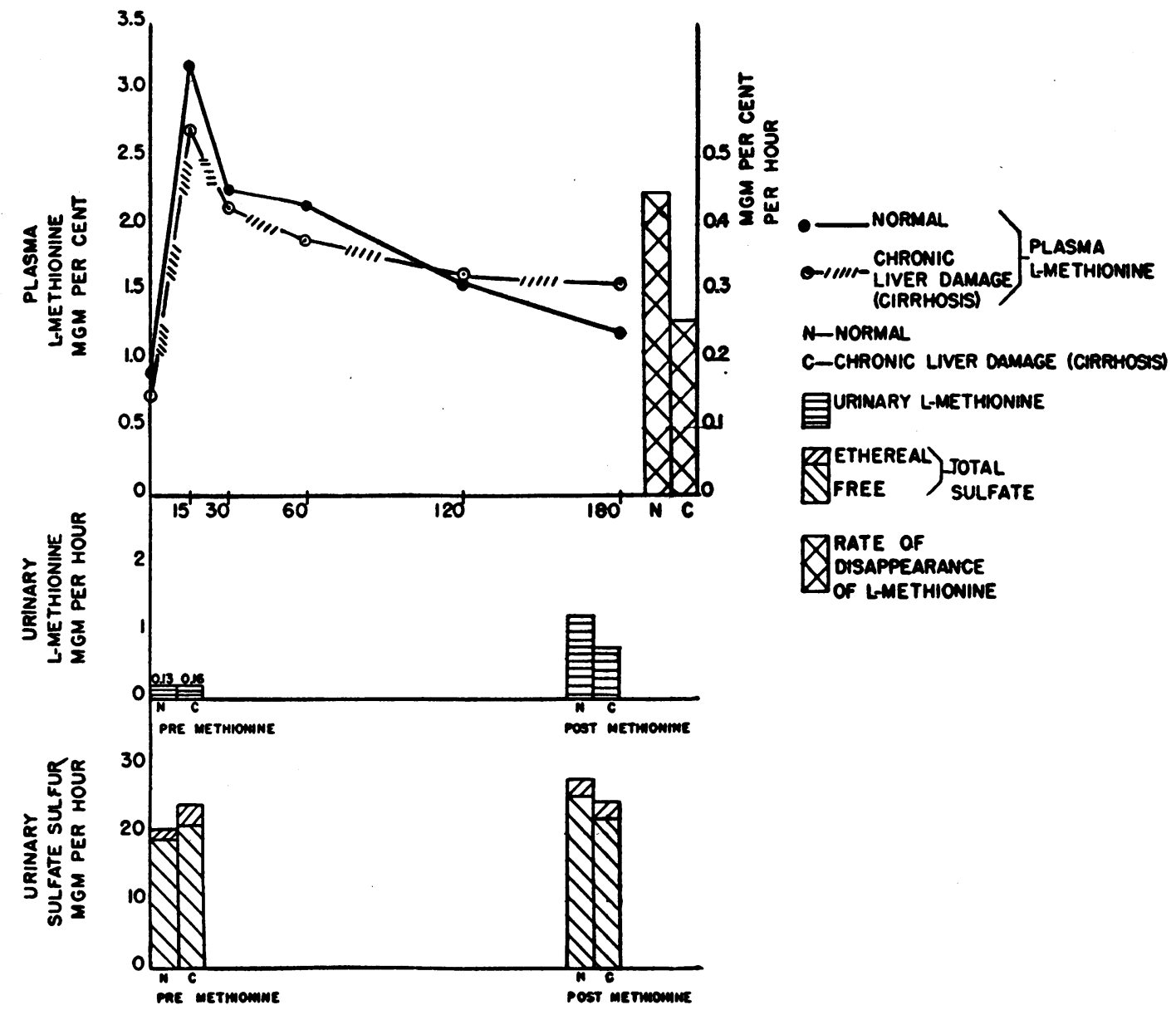

Fig. 6. Comparison of L-Methionine and Sulfate Values in Normal Individuals, and in Patients with Chronic Liver Damage (Cirrhosis)

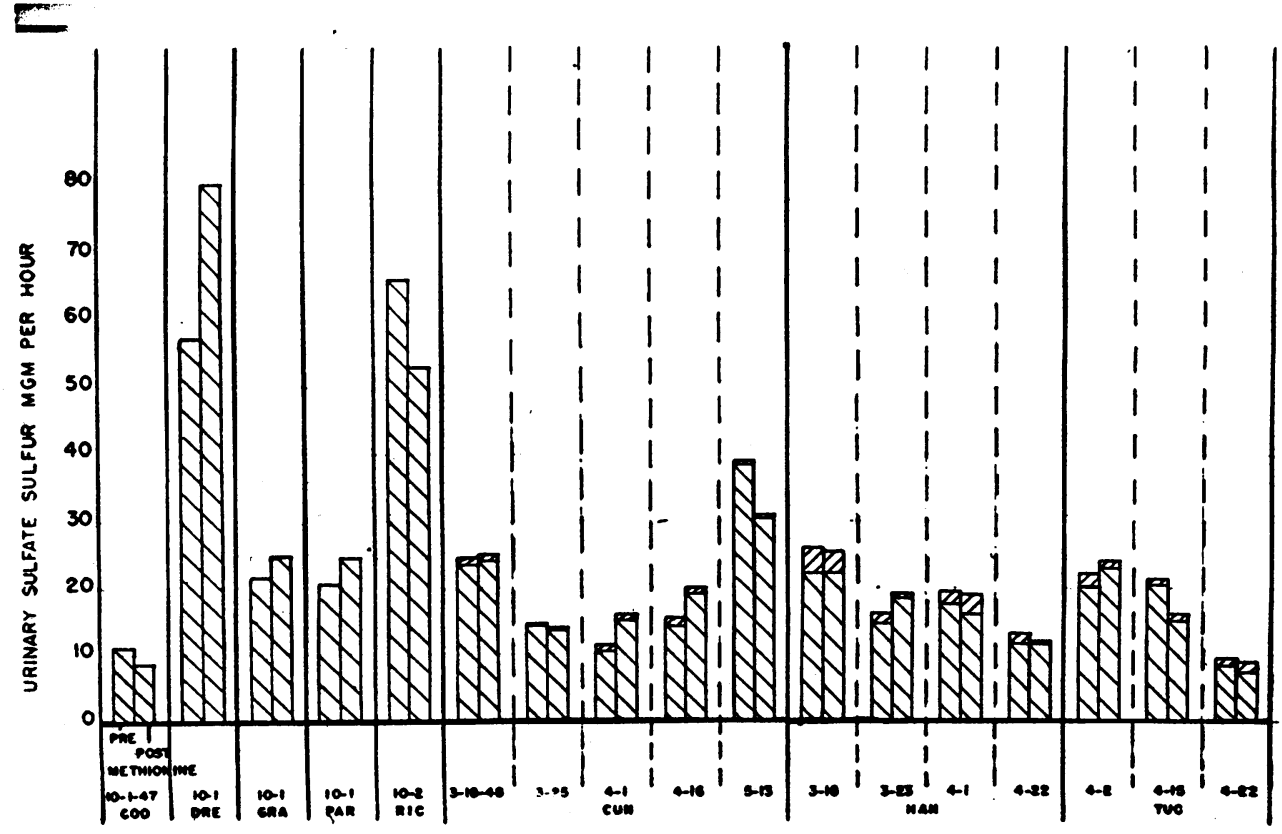

Fig. 7. Uninary Sulfate, Pre- and Post-Methionine, in a Group of Patients with Chronic liver Damage 

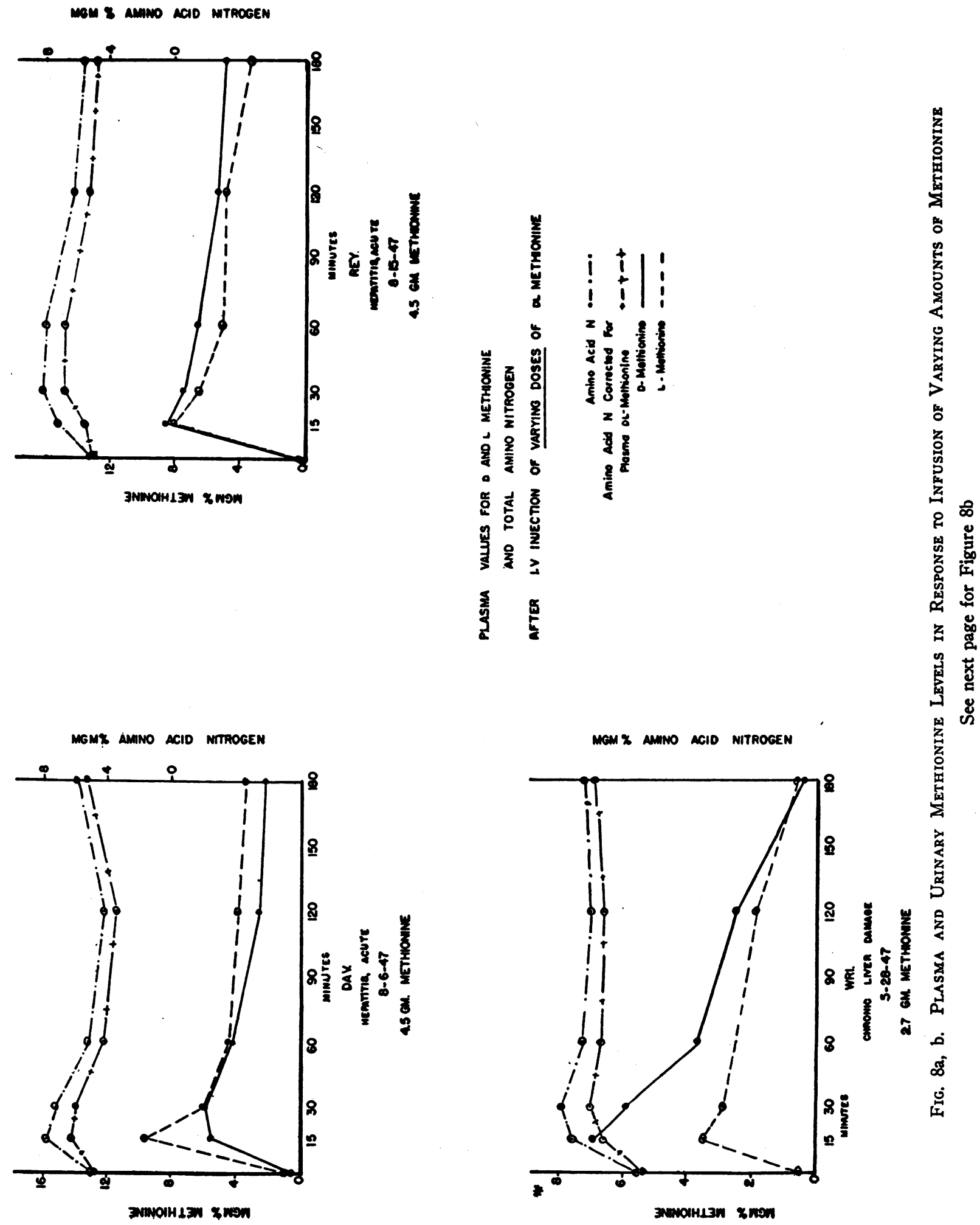
pre-methionine values, there was a sharp fall in the post-methionine sulfate in RIC (chronic hepatitis) and a sharp rise in DRE. Patient RIC has made some response to therapy. DRE died of acute hepatic insufficiency about six weeks later.

\section{The use of larger doses of methionine}

The original selection of 1.5 grams of $D L$ methionine as the intravenous dose was highly arbitrary based upon no more profound reason than that the water solubility of the material at room
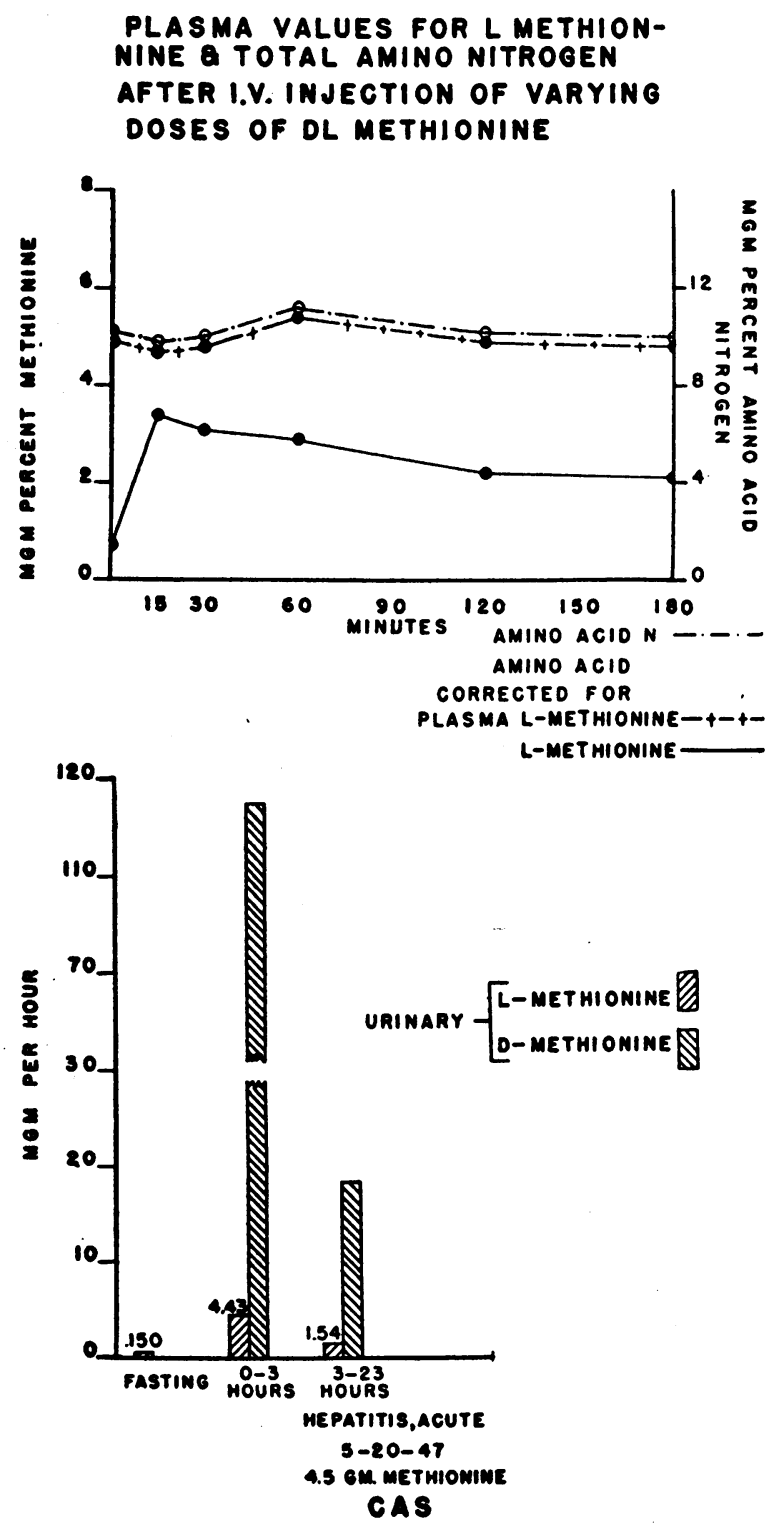

FIG. $8 b$

See legend, Fig. 8 a temperature was about 3 per cent, and that a $50-\mathrm{cc}$. syringe was the largest syringe with which one could conveniently work. That this dose proved to be an amount which answered many of our questions, has been a source of gratification.

A few patients have received larger dosages-up to 4.5 grams ( $150 \mathrm{cc}$. of a 3 per cent solution) given under the same conditions as those previously described for the smaller amount. The data so obtained indicate that the initial 15-minute values for $D$ - and $L$-methionine are roughly proportional to the dose; that the disappearance from the plasma of $L$-methionine follows the same pattern as in the case of the smaller amount ; that there is not a significant increase in urinary $L$-methionine, but that the urinary $D$-methionine content is roughly proportional to the amount administered (Figures $8 \mathrm{a}$ and $8 \mathrm{~b}$ ). The net result of this small number of observations was to assure us that a larger, less convenient dose had no physiological advantage.

\section{DISCUSSION}

The purpose of this paper is to present certain fundamental observations on methionine metabolism, particularly insofar as this subject is related to normal and abnormal liver physiology in the human subject. The possible clinical implications of these data will be considered in a separate communication, in which correlation of these procedures and of certain standard tests of liver function will be stressed.

\section{Urinary excretion}

One would expect that the natural isomer of an essential amino acid would have a high priority rating in terms of renal tubular reabsorption. Such is the case in man with $L$-methionine as adequately demonstrated in all subjects studied and with all doses used. Furthermore, that the renal physiology of $L$-methionine is not significantly altered by acute or chronic liver damage is likewise well demonstrated, despite the major differences in the rate of "utilization" of the $L$-isomer, as evidenced by blood studies, in many patients with liver damage. Reports on urinary methionine content $(11,12)$, therefore probably have no significance in terms of normal or abnormal liver physiology. 


\section{The fate of D-methionine}

The interpretation of the data obtained on plasma and urinary $D$-methionine is difficult. It is apparent that rapid and considerable renal excretion occurs in all instances. Such data as are available on urinary methionine in patients who received the 4.5 gram dose of $D L$-methionine, suggest that a mathematical relationship does exist between the size of the dose of the $D$-isomer and the urinary content of this material, as contrasted to the natural isomer. Whether biological tubular reabsorption exists is still to be determined. In any event, it appears that the rate of disappearance from the plasma, while, on the average, more rapid than that of the natural isomer, cannot be accounted for on the basis of renal excretion alone, nor can it be accounted for on the basis of total catabolism of the methionine molecule. Hence, anabolism of $D$-methionine must occur. Whether such metabolism initially requires deamination followed by transamination to the $L$-isomer is unknown to us at the present time. The presence of a specific enzyme ( $D$-amino acid oxidase) in liver and kidney which initiates such a reaction is well known. Quantitation of keto acid production and of other metabolites, now in progress, may help to throw further light on this problem.

\section{Fasting plasma methionine}

An additional factor which deserves emphasis in regard to $L$-methionine is the high fasting level noted in a few patients with acute hepatitis and during the acute phase of chronic liver damage, e.g., Patient SMI (Figure 3). As the disease subsides, the elevated level returns to normal well before a normal rate of utilization is achieved. The analogy to the behavior of blood dextrose in diabetes is obvious. Work now in progress indicates that very high fasting plasma $L$-methionine levels in cirrhotics may be found for several days after the discontinuance of oral methionine.

\section{L-methionine metabolism}

The evidence in support of the interpretation of the rate of fall during the 30 to 180 -minute period is also worthy of further discussion, as such an interpretation is still to be proved. That the rapid fall during the 15 to 30 -minute period is largely referable to diffusion, is well evidenced by the plasma-interstitial (edema) fluid values obtained in patient HAN (Figure 2). It is also apparent from the same data that once this initial diffusion has occurred, equilibrium is maintained between these two phases of body fluid. As already noted no phase of the $L$-methionine plasma curve is referable to urinary excretion and hence only two interpretations of the 30 to 180 -minute fall appear to be possible, viz., metabolism or intracellular storage. It would appear unlikely that cellular storage of methionine, as such, would be influenced significantly by impaired liver function and thus only one interpretation remains, viz., utilization (anabolism or catabolism).

Because of the fortuitous presence of sulfur in the methionine molecule, and the mandatory excretion of this sulfur as urinary sulfate when the amino acid is metabolized, one can speak with more assurance of catabolism than of anabolism. From the urinary sulfate data which we have so far obtained, one can say that no significant catabolism of the infused methionine occurs over the threehour post-infusion period, with a single exception (DRE, Figure 7). That this exception occurred in a man who died of hepatic insufficiency a few weeks later is probably of considerable importance. This man, to our initial discomfiture, had a normal disappearance rate. In retrospect, we believe that this finding was referable to two factors-the excessive rate of catabolism, and the presence of a huge amount of ascites at the time the test was done. Since the majority of cirrhotics with ascites, but without evidence of excessive catabolism, have had abnormally slow rates, it is probable that the catabolic factor was of more importance in the production of a "normal" rate of disappearance than was the increased interstitial fluid volume.

"False normal" rates of disappearance of the $L$-isomer have also been noted in a few instances in men who have had abnormally high fasting levels. It may be that one should not attempt to evaluate the utilization rate until the fasting level has returned to normal.

One further word concerning anabolism-catabolism: the small amount of urinary sulfate data which have so far accumulated in normal individuals suggests that little of the administered methionine is catabolized during the first three 
hours. This could be construed as an anabolic effect. Nitrogen-sulfate ratio determinations now in progress should support or refute this impression. A number of the acute and chronic liver patients have excreted less urinary sulfate after the methionine than in the basal, pre-methionine period. It is our present feeling that this serves as a significant index of anabolic effect. Confirmation of this impression, and of other of our present interpretations of the data presented, await the accumulation of further pertinent information in the patients here presented, and in others currently under study.

\section{SUMMARY AND CONCLUSIONS}

1. Normal human subjects remove intravenously administered methionine from the plasma at a predictable rate.

2. This rate of removal is impaired in many individuals with liver damage.

3. On the basis of the evidence presented, it seems probable that the rate of removal is synonymous with the rate of utilization (anabolism and/ or catabolism).

4. Urinary sulfate may serve as an anabolic/ catabolic index.

5. Under all conditions studied, the urinary excretion of $L$-methionine is negligible, while that of $D$-methionine is considerable.

\section{BIBLIOGRAPHY}

1. (a) Van Slyke, D. D., and Meyer, G. M., The fate of protein-digestion products in the body. III. The absorption of amino acids from the blood by the tissues. J. Biol. Chem., 1913, 16, 197.

(b) Van Slyke, D. D., and Meyer, G. M., The fate of protein-digestion products in the body. IV. The locus of chemical transformation of absorbed amino acids. Ibid., 213.

2. Jastrowitz, H., Versuche über Glykokollabbau bei Lebershädigungen. Arch. f. exp. Pathol. u. Pharmakol., 1908, 59, 463.

3. Bernhart, F. W., and Schneider, R. W., A new test of liver function, the tyrosine tolerance test. Am. J. M. Sc., 1943, 205, 636.

4. Witts, L. J., Observations on the metabolism of amino acids in health and disease. Quart. J. Med., 1929, 22, 477.

5. Kirk, E., Amino Acid and Ammonia Metabolism in Liver Disease. Levin and Munksgaard, Copenhagen, 1936.

6. Snell, E. E., The microbiological assay of amino acids, in Advances in Protein Chemistry, Anson, M. L., and Edsall, J. Academic Press, New York, 1945, Vol II, p. 85.

7. Harper, H. A., Kinsell, L. W., and Barton, H. C., Plasma $L$-methionine levels following intravenous administration in humans. Science, 1947, 106, 319.

8. Kinsell, L. W., Harper, H. A., Barton, H. C., Michaels, G. D., and Weiss, H. A., Rate of disappearance from plasma of intravenously administered methionine in patients with liver damage. Science, 1947, 106, 589.

9. Fiske, C. H., The determination of inorganic sulfate, total sulfate, and total sulfur in urine by the benzidine method. J. Biol. Chem., 1921, 47, 59.

10. Danielson, I. S., Amino nitrogen in blood and its determination. J. Biol. Chem., 1933, 101, 505.

11. Homburger, F., The urinary excretion of methionine in liver disorder. Am. J. M. Sc., 1946, 212, 68.

12. Wheeler, J. E., and György, P., Studies of urinary excretion of methionine by normals and by patients having liver disease. Am. J. M. Sc., 1948, 215. 267. 